\title{
Doppler assessment of the interventricular pressure drop in patients with ventricular septal defects
}

\author{
A B HOUSTON, M K LIM, W B DOIG, J M REID, E N COLEMAN \\ From the Department of Cardiology and Department of Child Health, Royal Hospital for Sick Children, \\ Glasgow
}

SUMMARY Doppler ultrasound was used to assess the pressure drop between the ventricles in $10 \frac{8}{3}$ infants and children $(61<$ two years old) with a ventricular septal defect who underwent cardiage catheterisation. The pressure in both ventricles was measured at catheterisation in 103 patient3 either simultaneously through two catheters (41) or with a single catheter withdrawn across the septum or removed from one ventricle to the other (62). When pressure was measureg simultaneously with two catheters (41 patients) the peak to peak and instantaneous gradients showed a maximum difference of $20 \mathrm{~mm} \mathrm{Hg}$ with levels within $10 \mathrm{~mm} \mathrm{Hg}$ of each other in $3 \overrightarrow{6}$. Comparison of the difference in the gradients with the average of the measurements demonstratent a tendency for Doppler to underestimate the difference when it was high $(>50 \mathrm{~mm} \mathrm{Hg}$ ) and overestimate it when it was low. A Doppler estimate of a low pressure difference between the ventricles indicates pulmonary arterial hypertension and a high one low pulmonary artery pressure, but in the intermediate group Doppler is as yet not sufficiently sensitive to allow selectio of those patients who require further investigation and possible operation. Doppler ultrasound was found to be a sensitive method of detecting a very small ventricular septal defect. Thus althoug Doppler is a very useful means of assessing and following patients with a ventricular septal defecg further studies are required to determine its exact place in clinical practice.

In the assessment of the patient with a ventricular septal defect echocardiography simplifies the diagnosis, localisation, and measurement of the size of the defect and can provide information of prognostic value.' Accurate knowledge of its haemodynamic effects, however, cannot be obtained from imaging ultrasound and the decision on the need for surgical closure is still based largely upon cardiac catheterisation, with direct measurement of the pulmonary pressure, blood flow, and vascular resistance. Doppler ultrasound now provides a non-invasive means for obtaining physiological information and in those with a ventricular septal defect can potentially provide an estimate of the pulmonary pressure and flow.

Several different methods of measuring the pulmonary artery pressure with Doppler have been reported $^{2-4}$; one of the most simple is the use of the

Requests for reprints to Dr A B Houston, Royal Hospital for Sick Children, Yorkhill, Glasgow G3 8SJ.

Accepted for publication 20 January 1988
Bernoulli formula ${ }^{5}$ to measure the pressure drop from the left to the right ventricle. ${ }^{26}$ Theoreticallyo subtraction of this value from the systolic bloog pressure will provide an estimate of the right veno tricular pressure and thus of the pulmonary arter. systolic pressure. There are potential inaccuracies is this technique, in particular possible overestimatio of right ventricular pressure because of failure to align the beam with the flow jet, or underestimation because of differences between peak to peak an instantaneous gradients. While the initial reporte study did indeed show a tendency to underestimate the pressure gradient when it was high (and thus tof overestimate right ventricular pressure), ${ }^{2}$ two sub sequent ones did not confirm this. ${ }^{67}$ These wero studies of $52,{ }^{2} 28,{ }^{6}$ and $38^{7}$ patients who had cathet studies. Simultaneous right and left ventricular pres sures were measured in only 21 and it has not beef shown whether there is a significant difference bet? ween the peak to peak and instantaneous gradiente across a ventricular septal defect. Furthermore, onl $/ 8$ a total of 20 patients under two years were included io these studies and it is in this age range that the right 
ventricular pressure is likely to be high and accurate assessment of pulmonary artery pressure is most important to decide whether surgical closure is needed.

Further consideration of the technique is justified. Most critical is the accurate measurement of the pressure drop between the ventricles which we assessed in 109 patients with ventricular septal defect undergoing cardiac catheterisation; in 41 both ventricular pressures were measured simultaneously.

\section{Patients and methods}

We studied 109 patients aged 10 days to 17 years (mean 3 years 6 months); 61 were less than two years old, including 46 who were less than one year old. All underwent cardiac catheterisation, ventricular pressures being measured through fluid filled catheters attached to strain gauge transducers. In six the left ventricle could not be entered via the left atrium or through the ventricular septal defect and the physician decided not to attempt an arterial approach. The remaining 103 had situs solitus with atrioventricular and ventriculoarterial concordance; seven had multiple defects, nine a ductus arteriosus, 12 aortic valve disease, seven previous coarctation repair, four a pulmonary artery band, two an atrial septal defect, two mitral regurgitation, one significant pulmonary regurgitation, and two peripheral pulmonary artery stenosis. None had significant pulmonary stenosis or an atrioventricular canal. In these 103, ventricular pressures were measured either simultaneously through two catheters (41) or consecutively through a single catheter removed from one ventricle to the other or withdrawn across the ventricular septal defect from the left to right ventricle. The peak to peak pressure difference was measured in all and the instantaneous maximum gradient was measured in the $\mathbf{4 1}$ in whom simultaneous ventricular pressures were recorded.

In 85 patients the Doppler studies were performed at the time of catheterisation close to the time that withdrawal pressure was measured or simultaneously with pressure measurements in the 41 in whom the dual catheter system was used; in the others the study was performed within 24 hours of catheterisation. Doppler studies were performed with either a non-imaging continuous wave transducer with an Alfred Velocimeter interfaced to a Doptek spectrum analyser or with a Vingmed CFM 700 system with imaging and colour flow mapping. A cross sectional echocardiogram was performed to demonstrate the position of the septum and, in most patients, the site of the ventricular septal defect. Since the image gives only a guide to the optimal position and angulation for recording maximum velocity of flow, the entire left parasternal edge and the subxiphoid position were explored and the position and angulation of the probe were adjusted to obtain the maximum velocity signal.

The maximum frequency shift was measured from the Doppler signal; from which the maximum velocity in $\mathrm{m} / \mathrm{s}(\mathrm{V})$ was derived and the pressure drop in $\mathrm{mm} \mathrm{Hg}(\mathrm{P})$ estimated from the modified Bernoulli formula, $P=4 V^{2}$.

The Doppler estimate of the pressure drop was then compared with the following measured pressure differences: peak to peak in the total population of 103 patients; peak to peak in the 41 in whom simultaneous ventricular pressures were measured; and instantaneous maximum gradient in these 41 patients. In addition, in these 41 subjects the measured peak to peak and instantaneous maximum gradients were compared.

\section{Results}

MEASUREMENT OF THE PRESSURE GRADIENT Peak to peak gradients ( 103 patients) ranged from 0 to $135 \mathrm{~mm} \mathrm{Hg}$ and instantaneous ones (41 patients) from 0 to $155 \mathrm{~mm} \mathrm{Hg}$. The mean difference between the instantaneous maximum and peak to peak gradients (41 patients) was $5.9 \mathrm{~mm} \mathrm{Hg}$ and it was greater than $10 \mathrm{~mm} \mathrm{Hg}$ in only five of the 41 patients: $12 \mathrm{~mm} \mathrm{Hg}$ (76 v 64), $15 \mathrm{~mm} \mathrm{Hg}$ (92 v 77), $15 \mathrm{~mm} \mathrm{Hg}$ $(25 v 10), 16 \mathrm{~mm} \mathrm{Hg}(58 v 42)$, and $20 \mathrm{~mm} \mathrm{Hg}$ (155 v 135).

\section{DOPPLER STUDIES}

The maximum velocity flow signal was most frequently obtained from the mid to low left parasternal edge but on occasions it was found with the transducer in a more lateral or a subxiphoid position. The signal rose to its maximum velocity in approximately mid-systole, fell in late systole to its lowest level just after aortic valve closure, and usually, but not always, continued with low velocity flow through diastole (figs 1 and 2). In some instances an early to mid-diastolic velocity increase seemed to be caused by the superimposition of mitral valve flow. The signal was positive indicating flow towards the transducer in all but two patients, who had small muscular defects with the jet emerging from the septum at such an angle that it was directed away from the transducer. With a high velocity jet and a small defect it was not always possible to obtain a complete spectral envelope with a single transducer angulation; in some instances a slight alteration of the position of the transducer showed both the upstroke and downstroke (fig 3), though not necessarily simultaneously, and this permitted the maximum velocity to be measured. It was often important to increase the level 


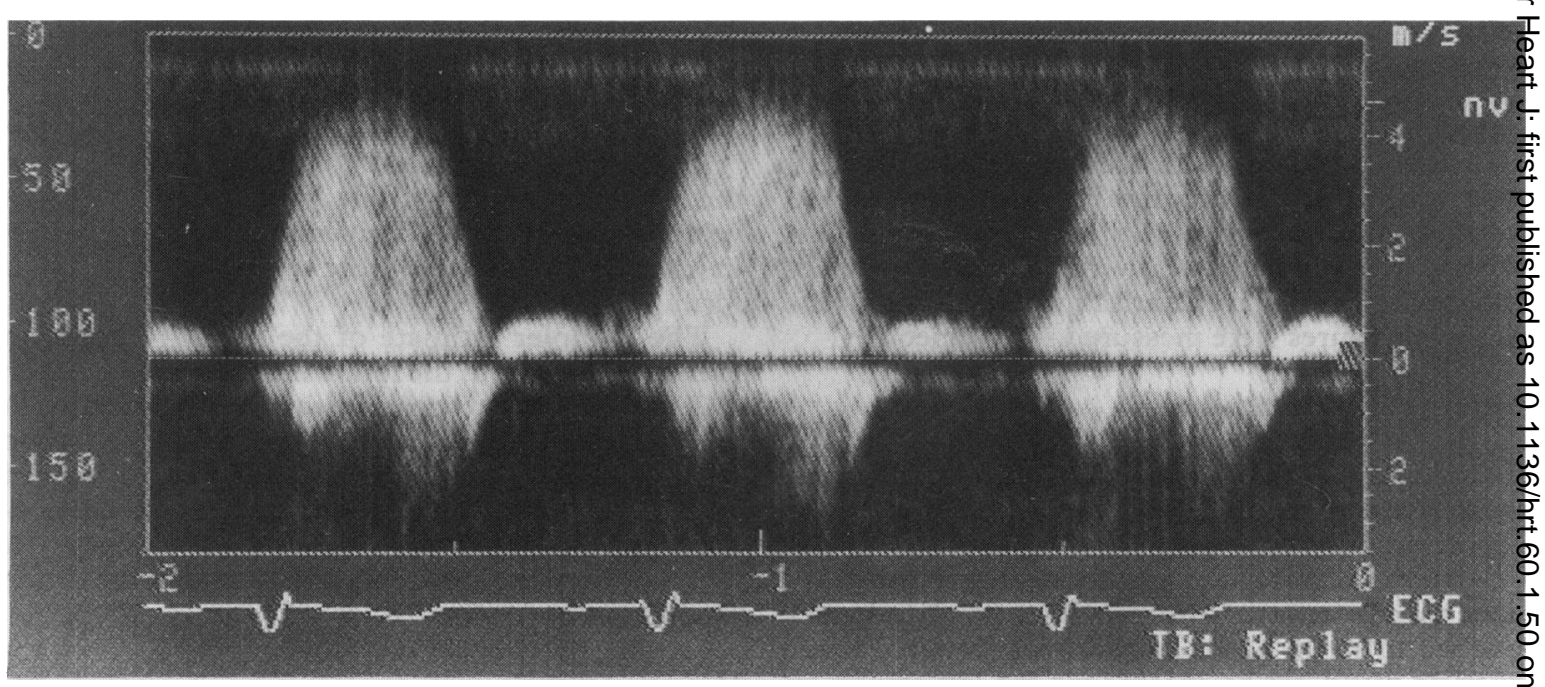

Fig 1 Spectral signal of flow through a ventricular septal defect in a patient with low pulmonary artery pressure and thus a large pressure difference between the ventricles. The maximum velocity of $4.25 \mathrm{~m} / \mathrm{s}$ is equivalent to a pressure difference of
$72 \mathrm{~mm} \mathrm{Hg}$.

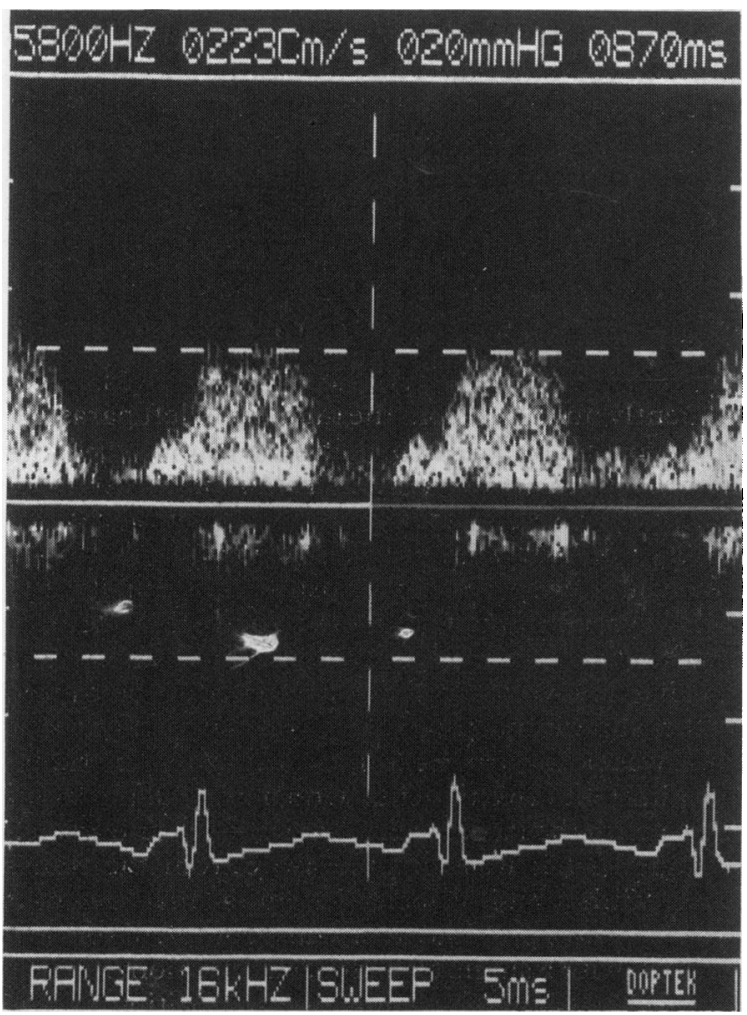

Fig 2 Spectral signal of flow through a ventricular septal defect in a patient with high pulmonary artery pressure and thus a small pressure difference between the ventricles. The marker line indicates the maximum velocity of $2.23 \mathrm{~m} / \mathrm{s}$, equivalent to a pressure difference of $20 \mathrm{~mm} \mathrm{Hg}$. of the high pass filter (maximum $5000 \mathrm{~Hz}$ with th $\overrightarrow{\mathrm{E}}$ Doptek spectrum analyser) to exclude hige amplitude, low frequency signals that can obscure the high frequency, lower amplitude signals that occur with a small ventricular septal defect.

Doppler gradients ranged from 0 to $110 \mathrm{~mm} \mathrm{Hg}$. Doppler signal of flow through the ventricular sept defect was not recorded in one infant with a peak to peak gradient of $30 \mathrm{~mm} \mathrm{Hg}$. This infant witi multiple defects was examined early in the study with a non-imaging transducer; in retrospect it seems likely that more experience and an assiduous examination technique would have permitted the signal to b recorded.

\section{COMPARISON OF DOPPLER AND MEASURED} GRADIENTS

The practical value of the technique was assessed by calculating the difference between the Doppler and measured gradients and comparing these values with the average value of the two measurements. ${ }^{8}$ The mean difference (2 SD) between the measurements was as follows: Doppler minus peak to peak for totaP population ( $n=103),-0.9(26.2)$ (fig 4); Dopples minus peak to peak measured with two cathetefs $(\mathrm{n}=41),-3 \cdot 1(24 \cdot 4)$; Doppler minus instantaneous maximum ( $n=41),-8 \cdot 3(24 \cdot 6)$ (fig 5).

Thus comparison of Doppler against peak to pea gradient in the total population shows that if the infant in whom no signal was obtained is excludedp the gradient was underestimated by $>15 \mathrm{~mm} \mathrm{Hg}$ i only those with a mean pressure drop of greater than $50 \mathrm{~mm} \mathrm{Hg}$; this underestimate was $>20 \mathrm{~mm} \mathrm{Hg}$ i eight and between 15 and $20 \mathrm{~mm} \mathrm{Hg}$ in five (fig 4 . 

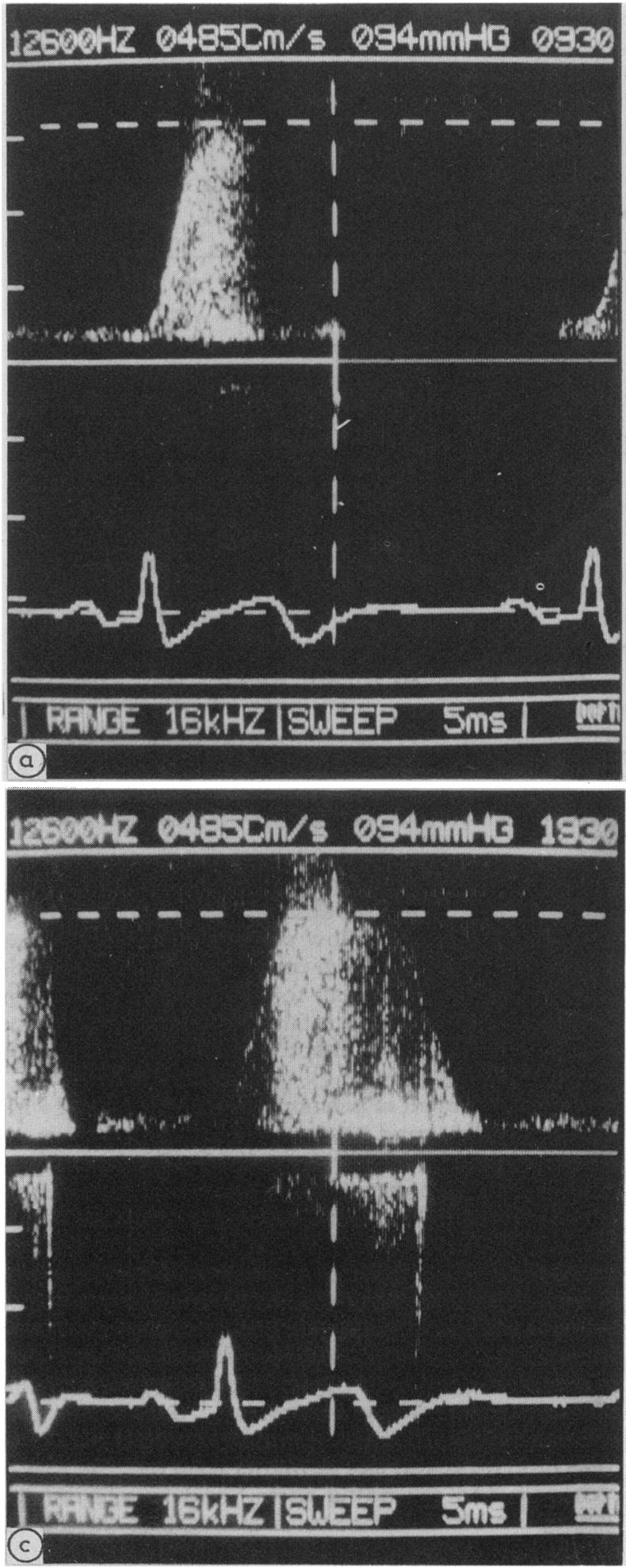

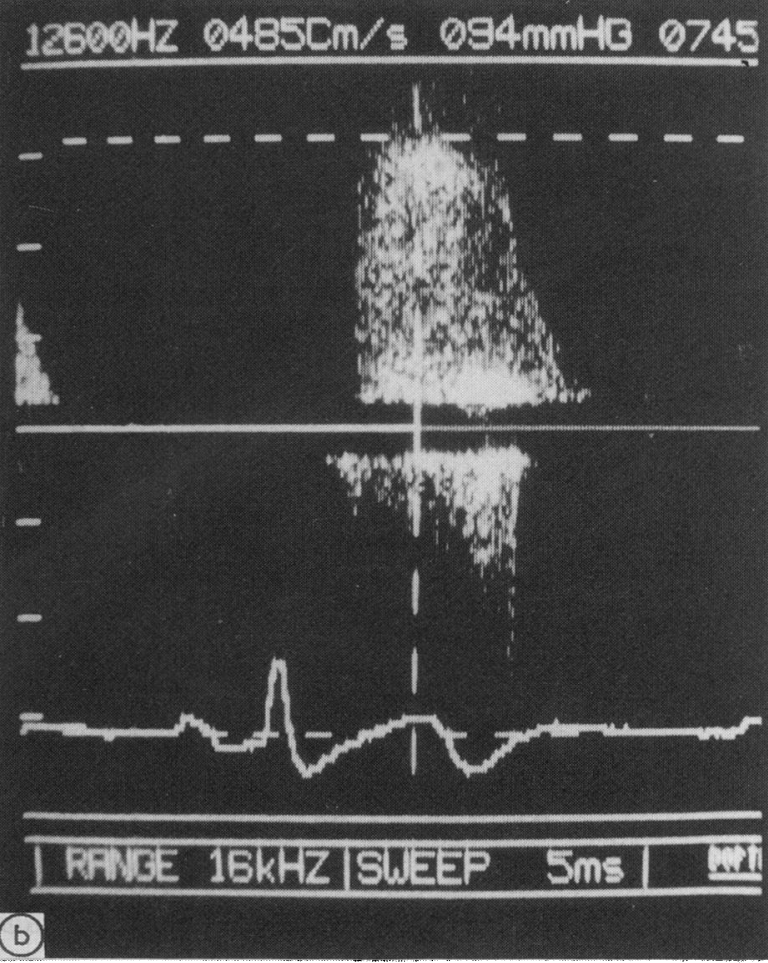

Fig 3 Spectral signals from a patient with a small ventricular septal defect and low pulmonary pressure. A complete, clear velocity envelope is difficult to obtain. The initial flow is best shown in (a) and later flow in (b); adjustment of the transducer produced $(c)$, demonstrating the complete envelope but with outlines of low intensity.

They had small defects and since the direction of the jet is likely to vary with cardiac contraction it is possible that the Doppler beam was not optimally aligned with it, and thus underestimated the pressure difference. On the other hand, although an overestimation $>15 \mathrm{~mm} \mathrm{Hg}$ occurred in 10, this was $>20$ $\mathrm{mm} \mathrm{Hg}$ in only two and between 15 and $20 \mathrm{~mm} \mathrm{Hg}$ in eight. This overestimation may be due to differences between instantaneous and peak to peak gradients or non-simultaneous recording. Simultaneous ventricular pressure measurements were made in only two of these patients; Doppler showed $80 \mathrm{~mm} \mathrm{Hg}$ (with a peak to peak measurement of $64 \mathrm{~mm} \mathrm{Hg}$ and an instantaneous one of $73 \mathrm{~mm} \mathrm{Hg}$ ) in one, and 36 (against 20 and $23 \mathrm{~mm} \mathrm{Hg}$ ) in the other. The studies of instantaneous gradients confirm the tendency to underestimate high gradients but in none was there an overestimation of $>15 \mathrm{~mm} \mathrm{Hg}$.

Of the 54 children with a measured peak to peak 


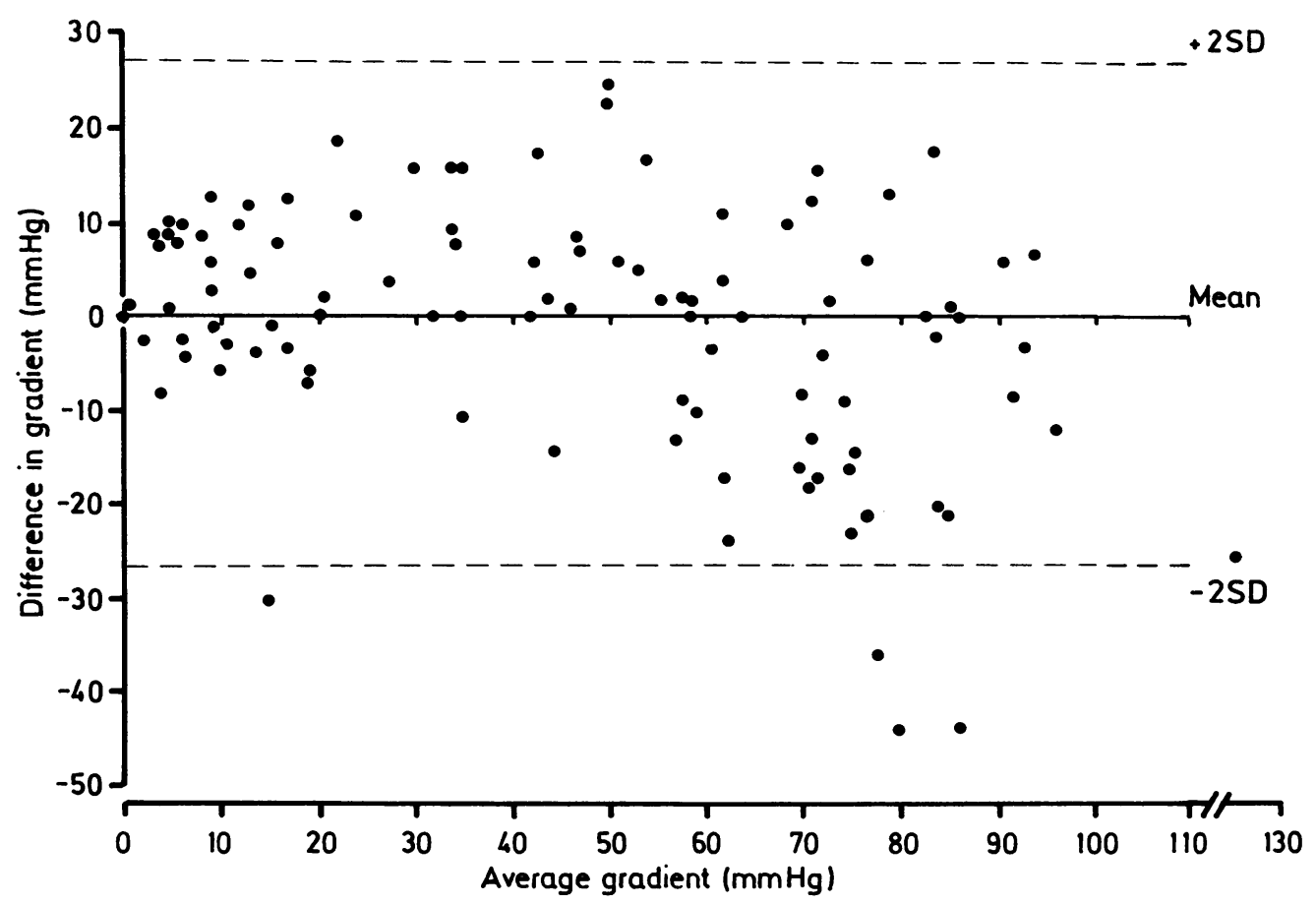

Fig 4 Comparison of the difference between pressure drops (Doppler minus peak to peak) against the average gradient in all 103 patients.

gradient of $<50 \mathrm{~mm} \mathrm{Hg}$, all but four were aged $<$ two. The pressure drop was underestimated by $>15$ $\mathrm{mm} \mathrm{Hg}$ in only the infant with multiple muscular ventricular septal defects in whom no definite flow signal was reported; since the right ventricular pressure would have been overestimated pulmonary hypertension would not have been missed. This child was seen early in the study and it is uncertain whether the failure to pick up the signal was because multiple defects were present or because of lack of experience in the technique. There was overestimation of the peak to peak pressure drop by $>15 \mathrm{~mm} \mathrm{Hg}$, with the possibility of underestimating pulmonary artery pressure, in seven of these 54, including one of the four with an average difference of $<25 \mathrm{~mm} \mathrm{Hg}$. None the less, in all 11 with an average difference of $<50$ $\mathrm{mm} \mathrm{Hg}$ in whom simultaneous studies against instantaneous gradient were made these were within $13 \mathrm{~mm} \mathrm{Hg}$ of each other.

\section{Discussion}

In patients with a ventricular septal defect echocardiography provides an anatomical diagnosis but is unable to give accurate information on haemodyn- amic function. Doppler ultrasound can confirm the site of a ventricular septal defect and, more importantly, measure blood flow velocity. Volumetric flow and pressure difference can be derived from this $\vec{\sigma}$ information. It may help to determine the need for surgical closure in the infant or young child or for prophylaxis against infective endocarditis in the 3 older patient.

The assessment of pulmonary artery pressure with Doppler ultrasound has been reported but as yet noo method has been accepted as reliable. Our experience suggests that in patients with a ventricular septalo defect a straightforward and repeatable method that may be suitable for routine use is the measurement of ${ }^{\circ}$ the pressure drop from the left to right ventricle. It is $\tilde{O}$ presumed that the systemic blood pressure approximates to the left ventricular systolic pressure ando that subtraction of the interventricular gradient fromo this should give the right ventricular, and in the absence of pulmonary stenosis, the pulmonary? arterial systolic pressure. The distal systolic blood pressure, however, can be higher than that in the lefto ventricle and ascending aorta, ${ }^{9}$ and in addition in young children accurate measurement of blood $\Omega$ pressure with an arm cuff can be difficult. 


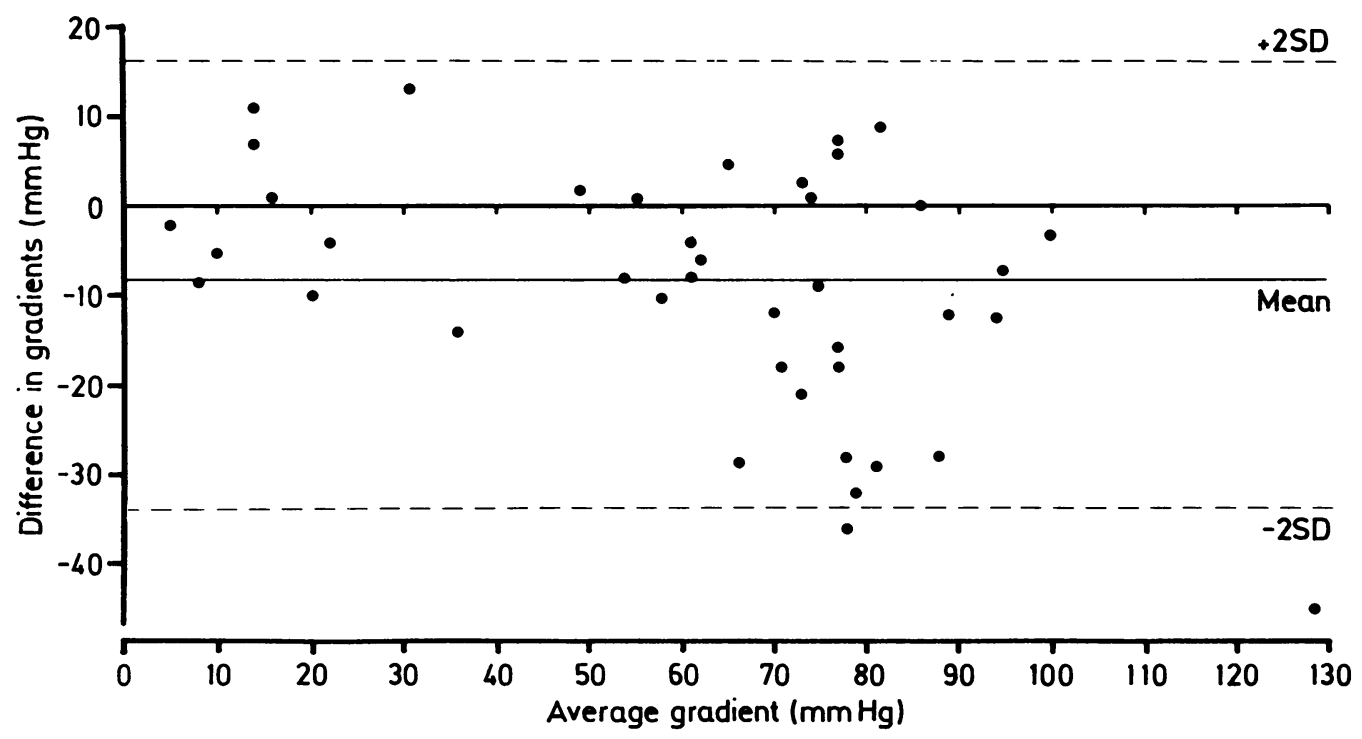

Fig 5 Comparison of the difference between pressure drops (Doppler minus instantaneous) against the average gradient in the 41 patients in whom pressures were measured simultaneously with two catheters.

This study has not attempted to compare arm and left ventricular pressures or accurately to calculate right ventricular pressure but has sought simply to assess the accuracy of Doppler measurement of the drop in pressure from the left to the right ventricle. There are several reasons why Doppler assessment of this might be inaccurate. If the beam is not aligned along the line of flow, the maximum blood velocity and thus the pressure drop will be underestimated with subsequent overestimation of the right ventricular and pulmonary arterial pressures, but pulmonary hypertension would not be missed. Doppler measures the instantaneous maximum and not the peak to peak gradient and, particularly in aortic stenosis, this can result in Doppler giving a significantly higher value than direct measurement of the peak to peak value. ${ }^{10}$ If there were similar differences in patients with a ventricular septal defect Doppler overestimation of the pressure drop could occur and the consequent underestimation of the pulmonary artery peak systolic pressure would have potentially dangerous results. This study has shown a relatively good correlation between the Doppler and measured gradients. As was found by Hatle and Rokseth ${ }^{2}$ there was a tendency to underestimate high gradients; this was not confirmed in the other studies ${ }^{67}$ Although the instantaneous pressure drops were higher than the peak to peak ones, this difference was small and not of clinical importance in the more important group with high pulmonary artery pressure.
The most valid comparison of pressure drops is obtained from Doppler studies performed simultaneously with pressure measurement from two catheters. The dual catheter results (fig 5) confirm the tendency of Doppler to underestimate the pressure drop in those with small defects and low right ventricular pressures. There is little evidence of overestimation of the drop in this group and indeed, for the instantaneous pressure drop the maximum overestimation by Doppler was only $13 \mathrm{~mm} \mathrm{Hg}$ (Doppler gradient $36 \mathrm{~mm} \mathrm{Hg}$ and catheter $23 \mathrm{~mm}$ $\mathrm{Hg}$ instantaneous and $20 \mathrm{~mm} \mathrm{Hg}$ peak to peak). Although only eight patients with an average gradient $<25 \mathrm{~mm} \mathrm{Hg}$ had dual catheter studies it seems likely that in general in this group the instantaneous pressure difference is little different from the peak to peak one.

Measurement of blood pressure in infants can present problems; the presumption that the systolic blood pressure in the arm equates with the left ventricular pressure adds a further error, calling into question the reliability of the measurement of right ventricular pressure.

It seems appropriate, however, to draw some conclusions from this study. If it is accepted that most patients who are less than two years old will have a systolic blood pressure of 65 to $110 \mathrm{~mm} \mathrm{Hg}$ (5th-95th centile $)^{11}$ and that those with a right ventricular pressure $<50 \%$ of the left will not require operation the following suggestions can be made. A Doppler recording of $<30 \mathrm{~mm} \mathrm{Hg}$ is likely 
to be associated with a right ventricular, and thus pulmonary artery pressure, of $>50 \%$ of systemic level, suggesting that operation is likely to be necessary, while a recording of $>55 \mathrm{~mm} \mathrm{Hg}$ suggests right ventricular pressure of $<50 \%$ of the systemic level and that operation can be deferred. But in patients with a Doppler record of between 30 and $55 \mathrm{~mm} \mathrm{Hg}$ Doppler as yet has not been shown to be sufficiently accurate to identify those who require operation and those who do not; in these patients it is appropriate to attempt to measure blood pressure and Doppler simultaneously, with the child sedated if necessary. While more information is awaited it is appropriate in this area of uncertainty to undertake further investigations, including cardiac catheterisation. In view of the small number of patients with pulmonary hypertension studied with two catheters further studies are necessary to confirm that there is not a clinically significant difference between peak to peak and instantaneous pressure drops in those with pulmonary hypertension and that this observation is unaffected by pulmonary vascular resistance and shunt size. Thus clinical decisions must take into account all the accepted clinical and non-invasive techniques in routine use.

In the symptom free older child with a systolic murmur at the lower left sternal edge clinical examination often provides reliable evidence of a ventricular septal defect; in some it can be difficult to differentiate between an innocent murmur and a small ventricular septal defect. In all our patients with a ventricular septal defect and normal right ventricular pressure Doppler demonstrated the jet, even where the defect was not evident on echocardiography and in one where the very small defect was barely shown on left ventricular angiocardiography. This is in keeping with previous findings ${ }^{2}$ and confirms that Doppler is an extremely sensitive technique for detecting the presence of flow through a small ventricular septal defect. This should allow a more effective approach to the management of the symptom free patient; the exclusion of a ventricular septal defect should prevent unnecessary follow up and antibiotic prophylaxis against infective endocarditis.

Although the extremes of high or normal right ventricular pressure are easily determined with clinical and other non-invasive studies in patients with a ventricular septal defect, we consider that Doppler measurement of the ventricular pressure difference is of practical value and significantly adds to the precision of the full non-invasive assessment. We are now convinced that it has become essential in the $c$ initial investigation of patients with a ventricular septal defect and is of particular value in the assess $-\frac{-7}{-}$ ment and subsequent follow up of the infant and young child.

\section{References}

1 Sutherland GR, Godman MJ, Smallhorn JF, Guiterrasw $P$, Anderson RH, Hunter S. Ventricular septa defects. Two dimensional echocardiographic and morphological correlations. Br Heart J 1982;47:316-8 28.

2 Hatle L, Rokseth R. Noninvasive diagnosis and assessment of ventricular septal defect by Doppler ultrasound. Acta Med Scand 1981;645:47-56.

3 Hatle L, Angelsen B. Doppler ultrasound in cardiology :physical principles and clinical applications. Philadel $\frac{c}{5}$ phia: Lea and Febiger, 1985:236-52.

4 Currie PJ, Seward JB, Chan KL, et al. Continuous $\overrightarrow{0}$ wave Doppler determination of right ventricularo pressure: a simultaneous Doppler-catheterisation study in 127 patients. J Am Coll Cardiol 1985;6 $750-6$.

5 Hatle L, Brubakk A, Tromsdal A, Angelsen B. Nonin- $\overline{\bar{O}}$ vasive assessment of pressure drop in mitral stenosisڤ by Doppler ultrasound. Br Heart J 1978;40:131-40. Ф

6 Murphy DJ, Ludomirsky A, Huta JC. Continuouswave Doppler in children with a ventricular septap $\overline{\overrightarrow{0}}$ defect: noninvasive estimation of interventricular pressure gradient. Am J Cardiol 1986;57:428-32.

7 Silbert DR, Brunson SC, Schiff R, Diamant S. Determination of right ventricular pressure in the presence of a ventricular septal defect using continuous wave Doppler ultrasound. J Am Coll Cardiol 1986;8 379-84.

8 Bland JM, Altman DG. Statistical methods for assess ing agreement between two methods of clinicab measurement. Lancet 1986;i:307-10.

9 Remington JW, O'Brien LJ. Construction of aortic flowo pulse from pressure pulse. Am J Physiol 1970; 218:437-47.

10 Currie PJ, Hagler DJ, Seward JB, et al. Instantaneous. pressure gradient: a simultaneous Doppler and duab catheter correlative study. J Am Coll Cardiol 1986;7:800-6.

11 Londe S, Goldring D. High blood pressure in children: problems and guidelines for evaluation and treat-o ment. Am J Cardiol 1976;37:650-7. 